\title{
The kirromycin gene cluster of Streptomyces collinus Tü 365 codes for an aspartate- $\alpha$-decarboxylase, KirD, which is involved in the biosynthesis of the precursor $\beta$-alanine
}

\author{
Kristina J Laiple, Thomas Härtner, Hans-Peter Fiedler, Wolfgang Wohlleben and Tilmann Weber
}

The Journal of Antibiotics (2009) 62, 465-468; doi:10.1038/ja.2009.67; published online 17 July 2009

Keywords: kirromycin; antibiotics; $\beta$-alanine; aspartate- $\alpha$-decarboxylase; PanD

In 1972, Heinz Wolf and Hans Zähner ${ }^{1}$ described the isolation and characterization of the narrow-host range antibiotic kirromycin from the actinomycete Streptomyces collinus Tü 365. Soon after its discovery, it was shown that the observed inhibitory effect of protein biosynthesis $^{2}$ is caused by the binding of kirromycin to the bacterial elongation factor $\mathrm{EF}-\mathrm{Tu},{ }^{3}$ a rare molecular target for antibiotics. Kirromycin binding inhibits the conformational shift that EF-Tu normally undergoes when GTP is hydrolyzed to GDP. ${ }^{4}$ This prevents dissociation of EF-Tu from the ribosomal complex and thus blocks translation. Owing to their target, 'elongation factor EF-Tu' kirromycin and related antibiotics such as aurodox ${ }^{5}$ or kirrothricin ${ }^{6}$ are also referred as elfamycins.

In our previous work, the kirromycin biosynthetic gene cluster was identified, isolated and analyzed. ${ }^{7,8}$ The linear carbon skeleton of kirromycin is synthesized by a highly complex hybrid type I polyketide synthase (PKS)/non-ribosomal peptide synthetase machinery encoded by the genes kirAI-kirAVI and kirB. The hybrid PKS/non-ribosomal peptide synthetase thereby combines trans-AT PKS architecture represented in the enzymes KirAI-KirAV with cis-AT architecture in the PKS KirAVI. Extender unit selection and loading are presumably carried out by two discrete acyltransferase enzymes, KirCI and KirCII, which are encoded in the gene cluster. ${ }^{8}$ Sequence analysis of the kirromycin biosynthetic gene cluster led to the proposal that $\beta$-alanine might be a building block for the typical pyridone moiety of kirromycin. This hypothesis was confirmed by feeding studies. Isotope-labeled $\beta$-alanine was efficiently incorporated at the expected positions in the kirromycin molecule. ${ }^{8}$

$\beta$-Alanine is an important primary metabolite that is required for vitamin $B_{5}$ (pantothenate) biosynthesis. Pantothenate itself is a pre- cursor of coenzyme A and phosphopantetheine, the prosthetic group of fatty acid or polyketide acyl carrier proteins and peptidyl carrier proteins of non-ribosomal peptide synthetases. In bacteria, $\beta$-alanine is mainly synthesized by the decarboxylation of aspartate catalyzed by aspartate- $\alpha$-decarboxylases (ADC), usually designated as PanD. ${ }^{9}$ ADC enzymes undergo a remarkable post-translational modification, which finally leads to the formation of an N-terminal pyruvoyl group that is required for catalytic activity. ${ }^{10}$ Owing to their essential function in pantothenate biosynthesis, ADC enzymes are ubiquitous in bacteria.

To our surprise, a putative $\mathrm{ADC}$, designated as $\mathrm{KirD}$, is encoded within the boundaries of the kirromycin biosynthetic gene cluster. KirD is highly similar to putative ADCs of other actinobacteria (for example, amino acid sequence identity $65 \%$, similarity $82 \%$ to PanD of Corynebacterium amycolatum SK46 (GenBank: ZP_03393336)). Although the site of post-translational processing at the $\mathrm{N}$ terminus is very high, the $\mathrm{C}$ terminus of the enzyme is less conserved and differs from other known streptomycete ADC sequences.

As typical primary metabolism genes within biosynthetic gene clusters are often accompanied by a second gene encoding an isoenzyme elsewhere in the genome, it was tested whether KirD is the only ADC present in S. collinus. Conserved regions were identified in a multiple sequence alignment of streptomycete PanD enzymes and degenerate primers PanDscreen5' (aagtcsaagatycaccg)/PanDscreen ${ }^{\prime}$ (acggcctgmgggctgcg) were deduced and used in a PCR with S. collinus genomic DNA. The resulting PCR products were subsequently cloned into the vector pJET (Fermentas, St Leon-Rot, Germany) and sequenced. Sequence analysis revealed the presence of a second putative ADC gene, designated as panD in S. collinus. Its gene product, PanD, forms a clade with other ADC (PanD) enzymes from strepto- 


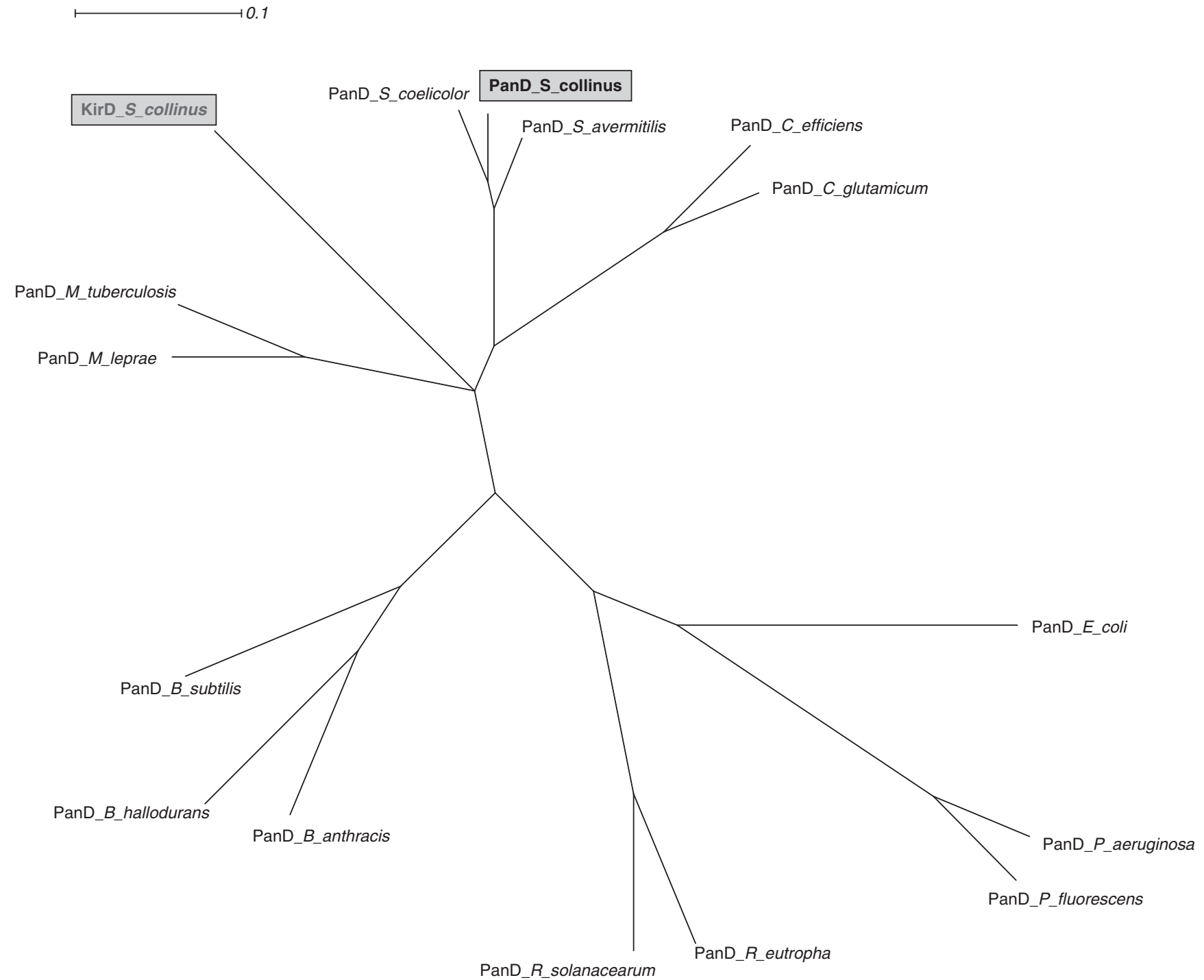

Figure 1 Consensus tree of aspartate- $\beta$-decarboxylases (PanD) of different bacteria. KirD and PanD of $S$. collinus Tü 365 are marked with a gray box. The sequences of the other PanD proteins were obtained from the UniProt database, aligned using muscle. ${ }^{18}$ The tree was calculated using Splitstree ${ }^{19}$ (BioNJ, 1000 bootstrap replicates, consensus tree).

mycetes, whereas KirD forms its own clade more closely related to enzymes from mycobacteria or corynebacteria than to enzymes from streptomycetes (Figure 1).

To test the involvement of KirD in kirromycin biosynthesis, the S. collinus mutant TL-D1 was constructed. As double crossover frequency is very low in $S$. collinus, the chromosomal kirD gene was inactivated by insertional inactivation with plasmid pTL-Dl, a pA18 derivative ${ }^{8}$ that contains a $265 \mathrm{bp}$ internal fragment of kirD and an ermE $E^{*}$-promoter to avoid polar effects on downstream genes. Correct integration of the plasmid was confirmed by Southern hybridization (Supplementary Figure S1, Supplementary information). HPLC-diode array $^{11}$ and HPLC-MS analysis of the extracts of S. collinus TL-D1 revealed that only traces of kirromycin were produced. These can be explained by residual activity of the primary metabolism equivalent to PanD, which may also be able to provide $\beta$-alanine for kirromycin biosynthesis. To confirm that this effect was due to the inactivation of kirD and not due to polar effects on essential downstream genes, exogenous $\beta$-alanine (final concentration $1 \mathrm{mg} \mathrm{ml}^{-1}$ ) was supplemented with the production broth. This led to a low but detectable increase in kirromycin production, although wild-type production levels were not reached. The absence of a full complementation may be caused by the fact that, in the mutant, the transcription of the downstream genes, the putative methyltransferase gene kirM and the putative acyltransferase gene kirCII, is under the control of the $\mathrm{ermE}^{*}$ promoter instead of their native promoter.

To further investigate the function of KirD in kirromycin biosynthesis, the kirD gene was amplified by PCR using the primers KirDoe5' (cgcgcaagcttaggaggatttaaaatgtaccgagagatgctcaagtcg; HindIII site+ Shine Dalgarno sequence) and KirDoe3' (gcgctctagatcacgcgt cgacggtgtcgccgcg; XbaI site) from cosmid $1 \mathrm{C} 24,{ }^{8}$ which carries the kirD region of the gene cluster, and cloned into pBlueskript II SK through the introduced restriction sites resulting in plasmid pTL-kirD1. Two $\beta$-alanine auxotrophic E. coli strains (E. coli SJ16 ${ }^{12}$ and E. coli DV9 ${ }^{13}$ ), which are mutated in panD and thus are unable to decarboxylate aspartate to $\beta$-alanine, were transformed with pTL-kirD1 or the empty vector, respectively. Their growth was monitored in a minimal medium with glucose and citrate as carbon sources. Neither strain grew in minimal medium if transformed with 
a
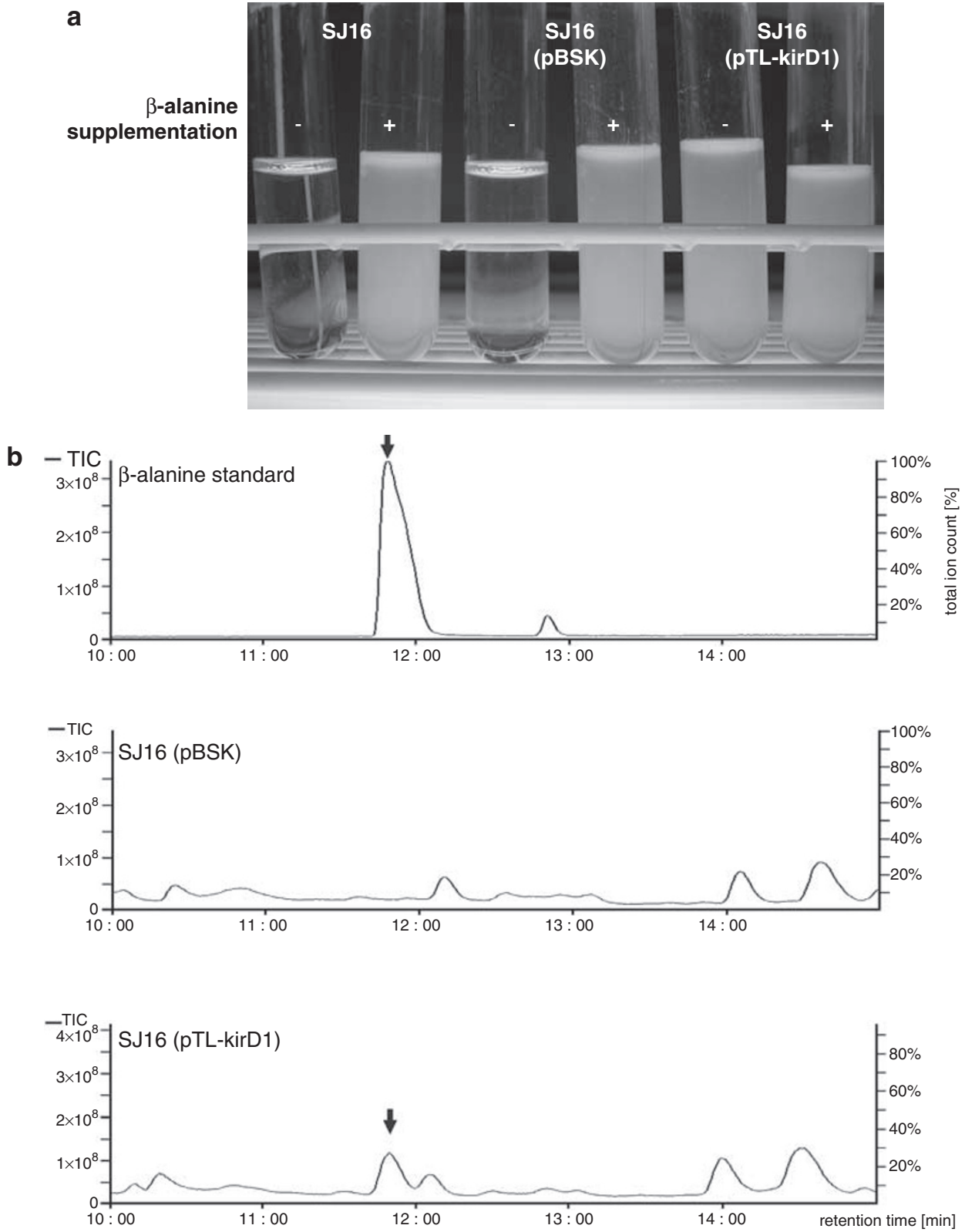

C $\beta$-alanine standard

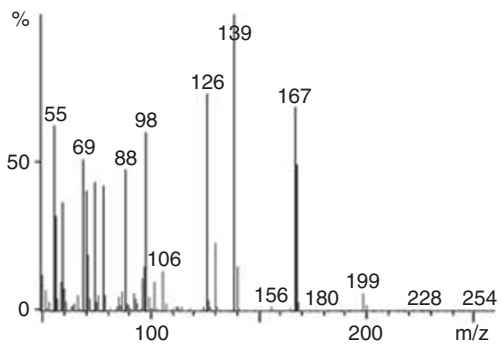

SJ16 (pTL-kirD1)

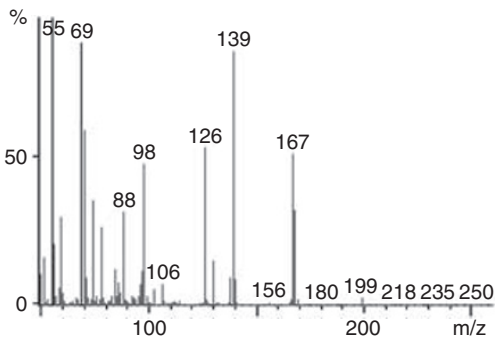

Figure 2 (a) Growth of E. coli SJ16, E. coli SJ16 (pBluescript) and E. coli SJ16 (pTL-D1). As a control, the different clones were also complemented with exogenously added $\beta$-alanine (end concentration $0.1 \mathrm{mg} \mathrm{ml}^{-1}$ ). (b) GC traces of a $\beta$-alanine standard and ADC assays using a crude extract from the control strain transformed with an empty pBluescript SK plasmid (SJ16 (pBSK)) and a crude extract of the kirD-expressing strain SJ16 (pTL-kirD1). $\beta$-alanine peak is marked with an arrow. The minimal differences of the retention times among the different samples are due to manual injection to the GC. (c) Mass spectrum of the peaks at retention time 11.8 min of the $\beta$-alanine standard and the ADC assay SJ16 (pTL-kirD1). 
the empty vector. Growth was only observed when transformants expressing kirD were analyzed (Figure 2a). This shows that $k i r D$ was successfully expressed and post-translationally processed in E. coli, and catalyzes the formation of $\beta$-alanine. Thus, a full complementation of the panD deficiency of E. coli SJ16 and DV9 was achieved by heterologously expressing kirD from the kirromycin biosynthetic gene cluster.

To finally prove that the effect observed in the growth experiments is due to the formation of $\beta$-alanine by KirD, assays for the in vitro conversion of aspartate to $\beta$-alanine were carried out. Crude protein extracts of E. coli SJ16 (pTL-kirD1) expressing the putative ADC from the kirromycin gene clusters cultivated in complex Luria Bertani medium and induced at an $\mathrm{OD}_{580}$ of 0.2 with $1 \mathrm{~mm}$ isopropyl- $\beta$-Dthio-galactopyranoside (IPTG) were prepared and used in slightly modified aspartate decarboxylase activity assays as described by Dusch et al. ${ }^{14} 2 \mathrm{~mm}$ aspartate was used as substrate and $50 \mu \mathrm{g}$ protein extract was applied per $200 \mu \mathrm{l}$ reaction volume. The reactions were carried out for $48 \mathrm{~h}$ at $37^{\circ} \mathrm{C}$ and stopped with the same volume of $3 \%$ perchloric acid and evaporated in a vacuum centrifuge. For gas chromatographic analysis, the samples were then hydrolyzed, derivatized with trifluoroacetic anhydride (TFAA), and finally resuspended in $100 \mu \mathrm{l} \mathrm{CH}_{2} \mathrm{Cl}_{2}$. Analysis was performed on a Shimadzu GC-17A gas chromatograph with coupled QP5000 mass detector on a Lipodex-E capillary column (diameter $0.25 \mathrm{~mm} \times 25 \mathrm{~m}$; film thickness $0.25 \mu \mathrm{m}$ ). By comparison with an $\beta$-alanine standard, it was shown that a substance with retention time $11.8 \mathrm{~min}$, which exactly corresponds to the retention time of $\beta$-alanine, is accumulated when using crude extracts of the complemented strain SJ16 (pTL-kirD1). No peak at this retention time was observed when using crude extracts of the control strain SJ16 carrying the empty pBluescript SK vector (Figure $2 \mathrm{~b}$ ). Mass spectrometric analysis of the substance eluting at $11.8 \mathrm{~min}$ revealed a mass peak of 199, which corresponds to the mass of the TFAA-derivatized $\beta$-alanine. The observed fragmentation pattern of both the accumulated metabolite and the $\beta$-alanine standard was identical, indicating that the accumulating substance in fact is $\beta$-alanine (Figure $2 c$ ). This finally proved that $\operatorname{KirD}$ is a functional aspartate- $\alpha$-decarboxylase catalyzing the conversion of aspartic acid to $\beta$-alanine.

The presence of KirD in the gene cluster indicates that $\beta$-alanine might be a limiting factor in kirromycin production. Therefore, the effect of $\beta$-alanine supplementation on kirromycin production with wild-type $S$. collinus was investigated under controlled conditions in a 51 fermenter using kirromycin production broth (soybean flour $10 \mathrm{~g}$, mannitol $10 \mathrm{~g}, \mathrm{CaCO}_{3} 5 \mathrm{~g}$, in 1.01 tap water, $\mathrm{pH} 7.3$ ). No effect was observed under optimized conditions in the fermenter.

Although the presence of typical 'primary metabolism' genes within antibiotic biosynthetic gene clusters has also been described for other biosynthesis pathways (for example, Shawky et al., 2007 15 ), only few experimental data concerning their function in antibiotic production exist. However, some reports have been published on the effects of inactivation or overexpression of primary metabolism genes that are involved in secondary metabolite precursor supply (for a review, see Olano et al., 2008 ${ }^{16}$ ) but are not encoded within secondary metabolite gene clusters. For example, in the actinorhodin biosynthesis of S. coeliocolor A3(2), the overexpression of acetyl-CoA carboxylase, which catalyzes the formation of the polyketide precursor malonyl-
CoA, led to a six-fold increase of actinorhodin yield, ${ }^{17}$ indicating a strong correlation between precursor availability and secondary metabolite production. This shows that maintaining precursor supply is important and may have an important function in the evolution of the gene clusters. Although the supplementation with $\beta$-alanine in rich media under controlled fermentation conditions did not lead to an increase of kirromycin production in S. collinus Tü 365, it is likely that this $\beta$-amino acid is still a limiting factor and its supply during secondary metabolite production may be required for efficient kirromycin production in the natural environment. To our knowledge, this is the first report of a cluster-encoded aspartate- $\alpha$-decarboxylase that is directly involved in providing $\beta$-alanine for antibiotic biosynthesis.

\section{ACKNOWLEDGEMENTS}

This work was supported by the German Federal Ministry of Education and Research (BMBF) with grant 0313805J (GenoMikPlus). We thank D Worbs for technical assistance.

1 Wolf, H. \& Zähner, H. Metabolic products of microorganisms. 99. Kirromycin. Arch. Microbiol. 83, 147-154 (1972).

2 Wolf, H., Zähner, H. \& Nierhaus, K. Kirromycin, an inhibitor of the $30 \mathrm{~S}$ ribosomal subunits function. FEBS Lett. 21, 347-350 (1972).

3 Wolf, H., Chinali, G. \& Parmeggiani, A. Kirromycin, an inhibitor of protein biosynthesis that acts on elongation factor Tu. Proc. Natl Acad. Sci. USA 71, 4910-4914 (1974).

4 Vogeley, L., Palm, G. J., Mesters, J. R. \& Hilgenfeld, R. Conformational change of elongation factor Tu (EF-Tu) induced by antibiotic binding. Crystal structure of the complex between EF-Tu.GDP and aurodox. J. Biol. Chem. 276, 17149-17155 (2001).

5 Liu, C. M., Williams, T. H. \& Pitcher, R. G. ${ }^{13} \mathrm{C}-$ NMR studies on the biosynthesis of aurodox (antibiotic X-5108). J. Antibiot. 32, 414-417 (1979).

6 Thein-Schranner, I., Zähner, H., Hoppe, H. U., Hummel, I. \& Zeeck, A. Metabolic products of microorganisms. 209 Kirrothricin, a new member of the kirromycin-group. J. Antibiot. 35, 948-956 (1982).

7 Weber, T., Welzel, K., Pelzer, S., Vente, A. \& Wohlleben, W. Exploiting the genetic potential of polyketide producing streptomycetes. J. Biotechnol. 106, 221-232 (2003).

8 Weber, T. et al. Molecular analysis of the kirromycin biosynthetic gene cluster revealed $\beta$-alanine as precursor of the pyridone moiety. Chem. Biol. 15, 175-188 (2008).

9 Cronan, Jr. J. E. ß-Alanine synthesis in Escherichia coli. J. Bacteriol. 141, 1291-1297 (1980).

10 Schmitzberger, F. et al. Structural constraints on protein self-processing in L-aspartate$\alpha$-decarboxylase. EMBO J. 22, 6193-6204 (2003).

11 Fiedler, H. P. Biosynthetic capacities of actinomycetes: 1. Screening for secondary metabolite products by HPLC and UV-visible absorbance spectral libraries. Nat. Prod. Lett. 2, 119-128 (1993).

12 Jackowski, S. \& Rock, C. O. Regulation of coenzyme A biosynthesis. J. Bacteriol. 148, 926-932 (1981).

13 Vallari, D. S. \& Rock, C. O. Isolation and characterization of Escherichia coli pantothenate permease (panF) mutants. J. Bacteriol. 164, 136-142 (1985).

14 Dusch, N., Pühler, A. \& Kalinowski, J. Expression of the Corynebacterium glutamicum pan $D$ gene encoding $L$-aspartate- $\alpha$-decarboxylase leads to pantothenate overproduction in Escherichia coli. Appl. Environ. Microbiol. 65, 1530-1539 (1999).

15 Shawky, R. M. et al. The border sequence of the balhimycin biosynthesis gene cluster from Amycolatopsis balhimycina contains $b b r$, encoding a StrR-like pathway-specific regulator. J. Mol. Microbiol. Biotechnol. 13, 76-88 (2007).

16 Olano, C., Lombo, F., Mendez, C. \& Salas, J. A. Improving production of bioactive secondary metabolites in actinomycetes by metabolic engineering. Metab. Eng. 10, 281-292 (2008)

17 Ryu, Y. G., Butler, M. J., Chater, K. F. \& Lee, K. J. Engineering of primary carbohydrate metabolism for increased production of actinorhodin in Streptomyces coelicolor. Appl. Environ. Microbiol. 72, $7132-7139$ (2006).

18 Edgar, R. C. MUSCLE: a multiple sequence alignment method with reduced time and space complexity. BMC Bioinformatics 5, 113 (2004).

19 Huson, D. H. SplitsTree: analyzing and visualizing evolutionary data. Bioinformatics 14, 68-73 (1998).

Supplementary Information accompanies the paper on The Journal of Antibiotics website (http://www.nature.com/ja) 